\title{
Extended hard X-ray emission from Vela X
}

\section{Fabio Mattana*1, Régis Terrier ${ }^{1}$, Diego Götz ${ }^{2}$, Laurent Bouchet ${ }^{3}$, Gabriele Ponti ${ }^{4}$, Teruaki Enoto $^{5}$, Maurizio Falanga ${ }^{6}$, Matthieu Renaud ${ }^{7}$, Isabel Caballero ${ }^{2}$, Simona Soldi $^{2}$ and S. Schanne ${ }^{2 \dagger}$}

${ }^{1}$ François Arago Centre, APC (Université Paris Diderot, CNRS/IN2P3, CEA/DSM, Observatoire de Paris), 13 rue Watt, F-75205 Paris Cedex 13, France

${ }^{2}$ CEA Saclay, DSM/IRFU/Service d'Astrophysique, F-91191 Gif-sur-Yvette, France

${ }^{3}$ CESR-Université de Toulouse/CNRS, 9 Av. du Colonel Roche, F-31028 Toulouse Cedex 04, France

${ }^{4}$ School of Physics and Astronomy, University of Southampton, Higheld, Southampton, SO17 1BJ, UK

${ }^{5}$ W. W. Hansen Experimental Physics Laboratory, Kavli Institute for Particle Astrophysics and Cosmology, Department of Physics and SLAC National Accelerator Laboratory, Stanford University, Stanford, CA-94305, USA

${ }^{6}$ International Space Science Institute (ISSI), Hallerstrasse 6, CH-3012 Bern, Switzerland

${ }^{7}$ Laboratoire de Physique Théorique et Astroparticules (LPTA), CNRS-UMR 5207, Université Montpellier II, F-34095 Montpellier, France

E-mail: fabio.mattana@apc.univ-paris7.fr

Vela $\mathrm{X}$ powered by the Vela pulsar is the best example of an evolved pulsar wind nebula, allowing to access the interaction with the supernova ejecta and the particle injection history. We present the spectrum of Vela $\mathrm{X}$ in the $20-300 \mathrm{keV}$ energy range as measured by IBIS/ISGRI and SPI, the main instruments onboard the INTEGRAL satellite. The apparent discrepancy between IBIS/ISGRI, SPI, and previous measurements is understood in terms of point spread function, pointing out a nebula more diffuse than previously thought. The presence of cooled electrons is also revealed by the spectral break measured including Suzaku data in the $1-10 \mathrm{keV}$ range. This picture is supported by the identification of a new structure in the energy band above $18 \mathrm{keV}$, extended along the NE/SW axis and partially coincident with the cocoon, the soft X-ray filament extending towards the centre of the remnant.

8th INTEGRAL Workshop "The Restless Gamma-ray Universe"

September 27-30 2010

Dublin Castle, Dublin, Ireland

\footnotetext{
* Speaker.

${ }^{\dagger}$ Based on observations with IBIS/ISGRI and SPI embarked on INTEGRAL, an ESA mission with instruments and science data centre funded by ESA member states (especially the principal investigator countries: Denmark, France, Germany, Italy, Switzerland, Spain), Czech Republic, and Poland, and with the participation of Russia and the USA. FM acknowledges the French Space Agency (CNES) for financial support.
} 


\section{Introduction}

Pulsar Wind Nebulae (PWNe) are the non-thermal bubbles inflated by the relativistic winds of rotation-powered pulsars. Recent high-resolution observations in X-rays and TeV Gamma-rays have allowed to understand the variety of the nebular morphologies as the result of an evolution driven by the confining medium [7]. In the first stage, a young pulsar and its nebula are embedded in a freely expanding supernova ejecta. The over-pressured PWN expands supersonically within the unshocked ejecta and appears roughly symmetrical with respect to the pulsar. When the SNR evolves into the Sedov-Taylor phase (after $\sim 10 \mathrm{kyr}$ since the pulsar birth), a reverse shock is formed within the ejecta. After a time of typically a few thousand years, the inward moving reverse shock collides with the boundaries of the PWN [18]. If the remnant is expanding in an inhomogeneus interstellar medium, the inward moving reverse shock reaches the PWN boundaries at different times, asymmetrically compressing the PWN and displacing the bulk of the electrons from the pulsar position [3]. The clearest example of a PWN in such a fairly evolved state is Vela X (Fig. 1, left panel).

Located at a distance of just $290 \mathrm{pc}$ [5], Vela $\mathrm{X}$ was discovered as a large $\left(\sim 2-3^{\circ}\right)$ radio nebula [17] embedded in the shell of the Vela supernova remnant, which has a radius of $3.5^{\circ}$. The nebula is powered by the Vela pulsar PSR B0833-45 (spin-down luminosity $\dot{E}=6.9 \times 10^{36} \mathrm{erg} \mathrm{s}^{-1}$, characteristic age $\sim 11 \mathrm{kyr}$ ). The most evident manifestation of the pulsar wind is found in the inner compact PWN observed by Chandra [8], which harbours a possible double torus and a complex jet-like structure. At larger scale, ROSAT found a X-ray filament extending $\sim 45^{\prime}$ south/south-west from the pulsar [13]. This so-called cocoon is thought to be generated by the reverse shock of the supernova remnant in the Sedov-Taylor phase. This explanation has been confirmed by the H.E.S.S. detection of extended $\mathrm{TeV}$ emission matching the X-ray cocoon [2], with the brightness peak significantly offset from the pulsar. The multiwavelength morphology and spectrum of Vela X, complemented by the recent GeV detections by AGILE [16] and Fermi [1], suggest two populations of electrons: a younger one, responsible of the $\mathrm{X}$-ray and $\mathrm{TeV}$ emissions, and an older one, more
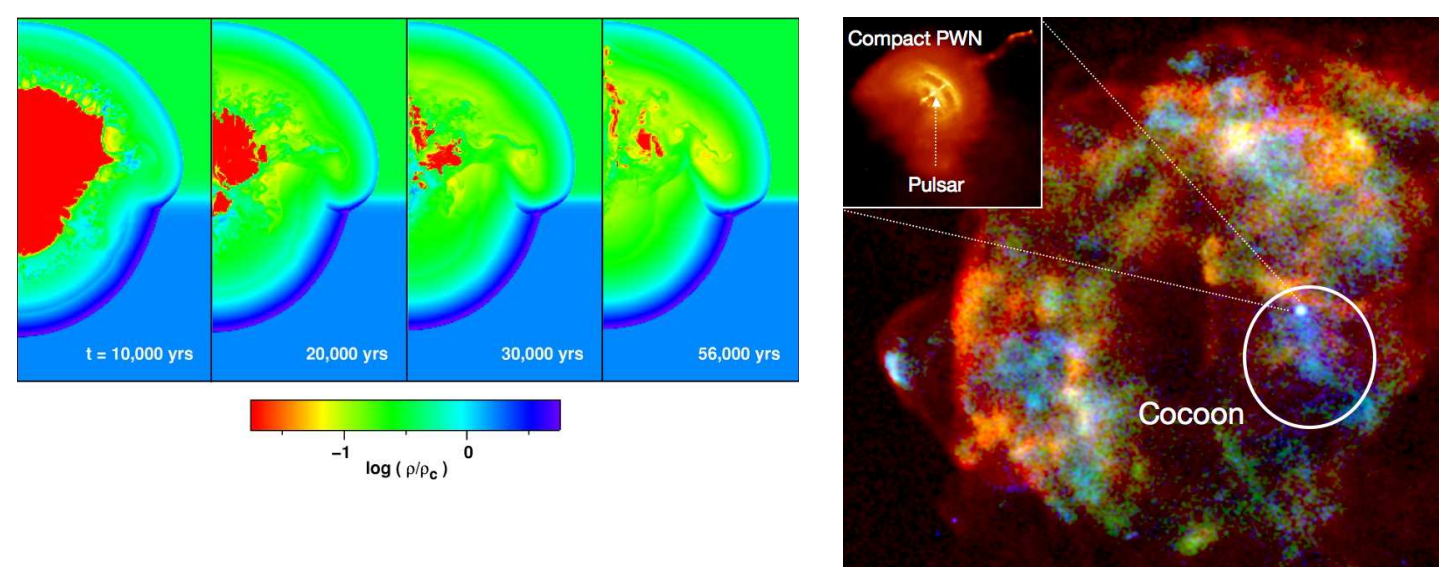

Figure 1: Left: simulated asymmetric crushing of a PWN inside a supernova remnant interacting with a non-uniform ambient medium. From [3]. Right: ROSAT/RASS X-ray image of the Vela supernova remnant, nebula and pulsar (red 0.1-0.4 keV, green 0.4-0.9 keV, and blue 0.9-2.4 keV). The nebula is composed by the inner compact PWN (inset: Chandra 1-8 keV [15]) and by the larger scale "cocoon". Vela pulsar lies in the centre of the inner PWN. 
diffuse and peaking in radio and $\mathrm{GeV} \gamma$-rays [4]. However, the location of the non-thermal electrons along the supernova remnant after the transit of the reverse shock is still not clear.

Simulations show that ejecta material can be turbulently mixed into the PWN by the reverseshock interaction, yielding a disrupted PWN. This is supported by ASCA observations suggesting possible non-thermal emission outside the cocoon [12], and by additional H.E.S.S. observations which revealed a more extended PWN in TeV gamma-rays [6]. INTEGRAL is the only observatory which can trace the most energetic electrons via their non-thermal X-ray emission. The angular resolution of IBIS/ISGRI on board the INTEGRAL observatory, unprecedented in the $20 \mathrm{keV}-1$ $\mathrm{MeV}$ range, combined with a large field of view, allows to adress the problem of the boundaries of the X-ray nebula. Here we report on the INTEGRAL identification of extended hard X-ray emission from Vela.

\section{INTEGRAL identification of extended hard X-ray emission}

We analyzed all public IBIS/ISGRI data within $12^{\circ}$ from the Vela pulsar, that is 1976 pointings collected between 2003-03 and 2008-07, for a total exposure time of 5.6 Ms. In the 18-40 keV mosaicked image (Fig. 2) we found a $85 \sigma$ point-like source. The radius of the point spread function (PSF) of $\sim 6^{\prime}$ encompasses the Vela pulsar and the structured inner nebula resolved by Chandra [8]. A hint of an extended feature is also apparent in the NE/SW direction, extending $\sim 50^{\prime}$ on both sides. The NE side is larger and more significant, whereas the thinner SW side is partially coincident with the ROSAT and H.E.S.S. cocoon (Fig. 2, second and third panels, respectively). The IBIS/ISGRI feature also matches the $2.5-10 \mathrm{keV}$ extended emission found by the Birmingham Spacelab 2 telescope [19] (Fig. 2, fourth panel). The individual pixels in the extended structure are at the $\sim 3-6 \sigma$ significance level, which does not represent a firm detection. However, such a large cluster of low-significance pixels is not observed in the rest of the image, and it is not reminescent of IBIS/ISGRI residual coding noise. A further evidence of extended hard X-ray emission from Vela $\mathrm{X}$ is provided by the spectral analysis.

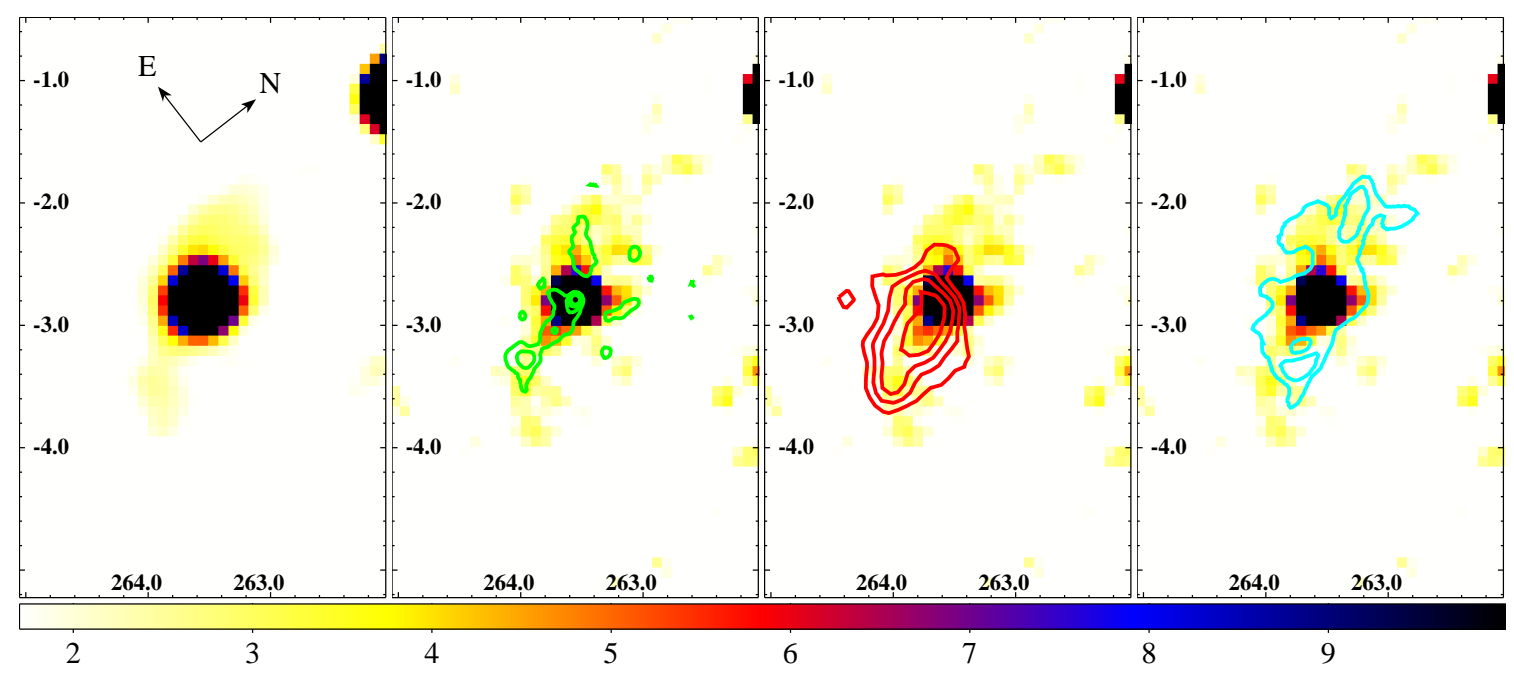

Figure 2: IBIS/ISGRI significance map in the $18-40 \mathrm{keV}$ range, Galactic coordinates. First panel: significance map smoothed with a 3 pixel $\left(\sigma \sim 7.5^{\prime}\right)$ Gaussian kernel. Other panels: unsmoothed significance map. Contours: ROSAT, 0.5-2 keV (second panel, green); H.E.S.S., VHE $\gamma$-rays above $1 \mathrm{TeV}$ (third panel, red); Spacelab 2, 2.5-12 keV (fourth panel, cyan). 

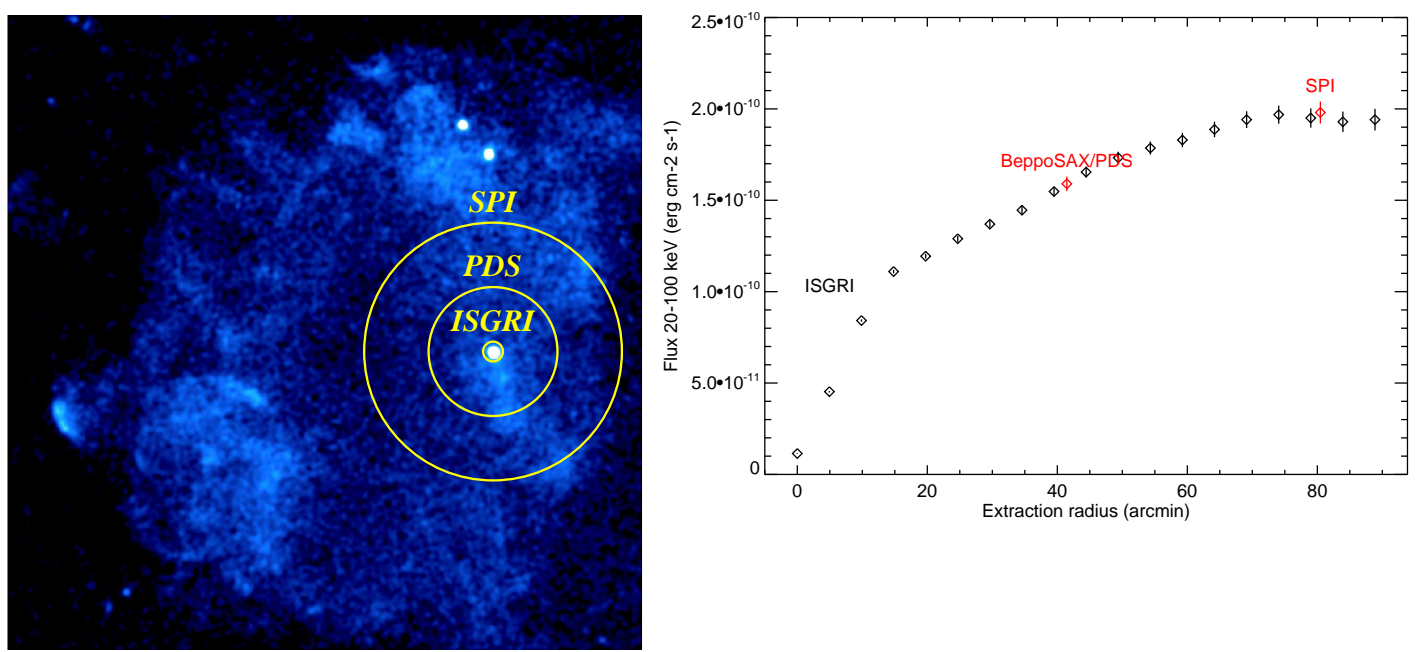

Figure 3: Left: ROSAT/RASS image in the 0.9-2.4 keV energy band. The PSF of different instruments used in this study is also shown. The non-thermal emission in the south-west is Puppis A. Right: integrated flux in the $18-40 \mathrm{keV}$ band at varying extraction radii (black diamonds), along with BeppoSAX/PDS and SPI fluxes in the same band. In this case the integration radius is given by their PSF $\left(6^{\prime}, 39^{\prime}\right.$, and $1.3^{\circ}$ for IBIS/ISGRI, BeppoSAX/PDS, and SPI respectively). The origin of the x-axis corresponds to the pulsar position. The third point of the IBIS/ISGRI integrated flux profile encloses the flux of the point-like source, that is the Vela inner compact nebula.

We extracted the IBIS/ISGRI spectrum of the point-like source from mosaicked images in narrow energy bands between 20 and $200 \mathrm{keV}$. It can be fitted with a single power law model with photon index $\Gamma_{\text {ISGRI }}=1.98 \pm 0.05$ and flux $F=(4.75 \pm 0.07) \times 10^{-11} \mathrm{erg} \mathrm{cm}^{-2} \mathrm{~s}^{-1}$ in the $20-40 \mathrm{keV}$ range. We also analyzed the SPI data from the same observations, and found a power law spectrum in the $20-300 \mathrm{keV}$ range with compatible photon index, $\Gamma_{\mathrm{SPI}}=2.15 \pm 0.15$, but higher flux, $F=(9.1 \pm 0.6) \times 10^{-11} \mathrm{erg} \mathrm{cm}^{-2} \mathrm{~s}^{-1}$ in $20-40 \mathrm{keV}$, with respect to IBIS/ISGRI. A joint fit of the IBIS/ISGRI and SPI data yields a compatible photon index, but it requires a factor 1.8 to recover the higher SPI flux. Such a discrepancy can not be due to an intercalibration factor, which in the case of IBIS/ISGRI and SPI is in the 0.8-1.2 range. Both the INTEGRAL photon indices are consistent with the one measured by BeppoSAX/PDS [10], $\Gamma_{\mathrm{PDS}}=2.01 \pm 0.05$, whereas the BeppoSAX/PDS flux lies between the IBIS/ISGRI and the SPI one. As shown in Fig. 3 , the IBIS/ISGRI, BeppoSAX/PDS, and SPI fluxes correlate with their respective PSF radii $\left(6^{\prime}\right.$, $39^{\prime}$, and $1.3^{\circ}$ ), suggesting that each instrument samples a different size of the nebula. Due to the coded mask deconvolution of IBIS/ISGRI, the reconstructed fluxes of an extended source of $60^{\prime}$ radius is lower than the real one by a factor $\sim 50$ [14]. Therefore, such a source with a flux of $4.3 \times 10^{-11} \mathrm{erg} \mathrm{cm}^{-2} \mathrm{~s}^{-1}$ (the difference between the SPI and ISGRI fluxes), would be measured by IBIS/ISGRI as low as $\sim 10^{-12} \mathrm{erg} \mathrm{cm}^{-2} \mathrm{~s}^{-1}$, close to to its sensitivity limit.

To support the hypothesis that an extended source is present but diluted in the coded mask deconvolution, we refined the analysis of the ISGRI data following the prescription for extended sources [14]. Then, we extracted the IBIS/ISGRI flux from concentric circles centred on the pulsar at extraction radii up to $100^{\prime}$ (Fig 3, right panel). After $15^{\prime}$, this integrated flux as a function of the extraction radius does not reach a plateau, as it would be expected from a point-like source, but slowly increases up to $\sim 60^{\prime}$. Most importantly, the IBIS/ISGRI flux nicely recovers the BeppoSAX/PDS and SPI fluxes at extracting radii comparable with their PSF. This confirms that Vela $\mathrm{X}$ is detected above $18 \mathrm{keV}$ and it is extended. 


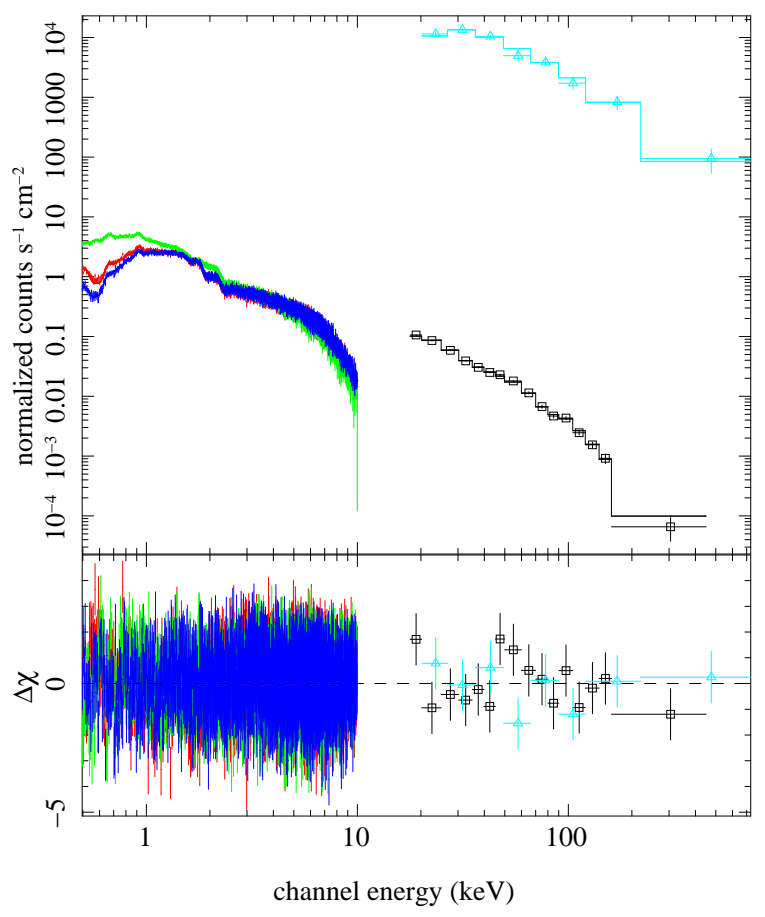

Figure 4: Combined Suzaku/XIS0-1-3 (red, green, and blue), IBIS/ISGRI (black), and SPI (cyan) spectra of the Vela PWN. Suzaku/XIS data were extracted in a circular region of $6^{\prime}$ radius centered on the pulsar to match the IBIS/ISGRI PSF. We modeled the pulsar contribution by fixing two black bodies and a power law, corrected by the interstellar absorption, with fixed parameters as measured by XMM-Newton [11]. A free constant factor was considered to fit the SPI data (see text). An absorbed non-equilibrium plasma emission model is also included to account for the thermal supernova remnant, as in [9].

\section{Combined IBIS/ISGRI and Suzaku/XIS spectral analysis}

The MECS and PDS on board BeppoSAX allowed to measure a spectral break at energy $12.5 \pm 1.5 \mathrm{keV}$ [10]. However, the different angular resolutions of the two instruments required the authors to rescale the PDS flux from $39^{\prime}$, its PSF, to $15^{\prime}$, the maximum extraction radius of MECS. With a much narrower PSF, INTEGRAL IBIS/ISGRI can be combined with Suzaku/XIS on the same extraction radius. Public XMM-Newton/EPIC-MOS data of the same field could not be used because they were taken in Small Window mode, to prevent the pile-up, which reduces the field of view to a fraction of the central chip. We then analyzed public available Suzaku data. Within an extraction circle of $6^{\prime}$ radius from the Vela pulsar position, the derived Suzaku/XIS spectrum shows a power law component with photon index $1.654 \pm 0.005$, and a 3-10 keV flux compatible with the MECS in the same region. To simultaneously fit the IBIS/ISGRI and XIS data, a spectral break is required at $27.4 \pm 3.5 \mathrm{keV}$, higher than the one derived by BeppoSAX on a larger region, which could suggest electron cooling in the propagating nebular outflow.

\section{Conclusions}

The main concern of this contribution was to establish the diffuse hard X-ray emission from Vela $\mathrm{X}$ as pointed out by INTEGRAL observations. The flux differences between IBIS/ISGRI, BeppoSAX/PDS, and SPI is explained by their different PSF, sampling different sizes of the PWN. The similar photon indexes found in the spectra of the three instruments suggest that the electron 
population radiating in hard X-rays has undergone a significant synchrotron burn-off. The detailed morphology of the hard X-ray nebula is still an open question. An indication of an extended feature is present in the IBIS/ISGRI image in the $18-40 \mathrm{keV}$ band. It is surprising to find that it extends mostly on the northern side, where the reverse shock scenario predicts the strongest compression. The physical implications and the details of these results will be presented in a forthcoming work.

\section{References}

[1] Abdo, A. A., et al. 2010, ApJ, 713, 146

[2] Aharonian, F., et al. 2006, A\&A, 448, L43

[3] Blondin, J. M., Chevalier, R. A., \& Frierson, D. M. 2001, ApJ, 563, 806

[4] de Jager, O. C., Slane, P. O., \& LaMassa, S. 2008, ApJL, 689, L125

[5] Dodson, R., Legge, D., Reynolds, J. E., \& McCulloch, P. M. 2003, ApJ, 596, 1137

[6] Dubois, F., et al. for the H.E.S.S. collaboratio, 2010, Proceeding of the $31^{\text {st }}$ Intenational Cosmic Ray Conference (ICRC 2009), Lodz, Poland

[7] Gaensler B. M., Slane P. O., 2006, ARA\&A, 44, 17

[8] Helfand, D. J., Gotthelf, E. V., \& Halpern, J. P. 2001, ApJ, 556, 380

[9] LaMassa, S. M., Slane, P. O., \& de Jager, O. C. 2008, ApJL, 689, L121

[10] Mangano, V., Massaro, E., Bocchino, F., Mineo, T., \& Cusumano, G. 2005, A\&A, 436, 917

[11] Manzali, A., De Luca, A., \& Caraveo, P. A. 2007, ApJ, 669, 570

[12] Markwardt, C. B., \& Ögelman, H. B. 1997, ApJL, 480, L13

[13] Markwardt, C. B., \& Ögelman, H. B 1995, Nature , 375, 40

[14] Renaud, M., et al. 2006, A\&A, 456, 389

[15] Pavlov, G. G., Teter, M. A., Kargaltsev, O., \& Sanwal, D. 2003, ApJ, 591, 1157

[16] Pellizzoni, A., et al. 2010, Science, 327, 663

[17] Rishbeth, H. 1958, Australian Journal of Physics, 11, 550

[18] van der Swaluw, E., Achterberg, A., Gallant, Y. A., \& Tóth, G. 2001, A\&A, 380, 309

[19] Willmore, A. P., Eyles, C. J., Skinner, G. K., \& Watt, M. P. 1992, MNRAS, 254, 139 